Supporting information for

\title{
Ultrafast Excited-State Dynamics of 6-Azauracil Studied by Femtosecond Transient Absorption Spectroscopy
}

\author{
Xinzhong Hua, Linqiang Hua, Xiaojun Liu* \\ State Key Laboratory of Magnetic Resonance and Atomic and Molecular Physics, Wuhan \\ Institute of Physics and Mathematics, Chinese Academy of Sciences, Wuhan 430071, P. \\ R. China \\ * Author to whom correspondence should be addressed. \\ e-mail: xjliu@wipm.ac.cn
}

Contents:

(1) Fig. 1S. Global fitting of the decay dynamics of 6-AU in acetonitrile at various wavelengths.

(2) Fig. 2S. Global fitting of the decay dynamics of 6-AU in methanol (a), ethanol (b), $n$-butanol (c) and water (d) at various wavelengths.

(3) Fig. 3S. Lifetime constants as a function of viscosity of different solvents. 


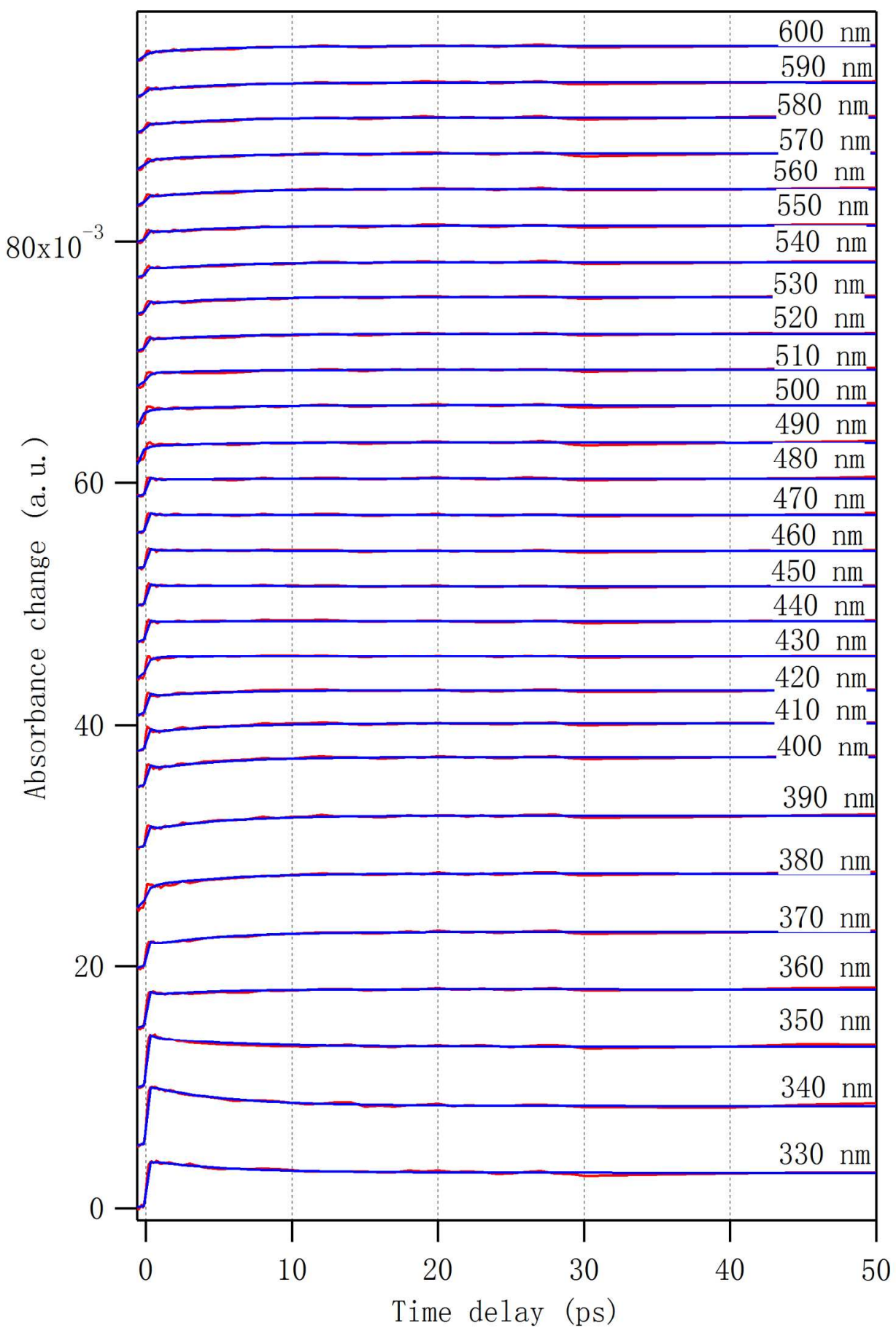

Fig. 1S Global fitting of the decay dynamics of 6-AU in acetonitrile at various wavelengths. Red line: experimental data; Blue line: fitting of the data. 


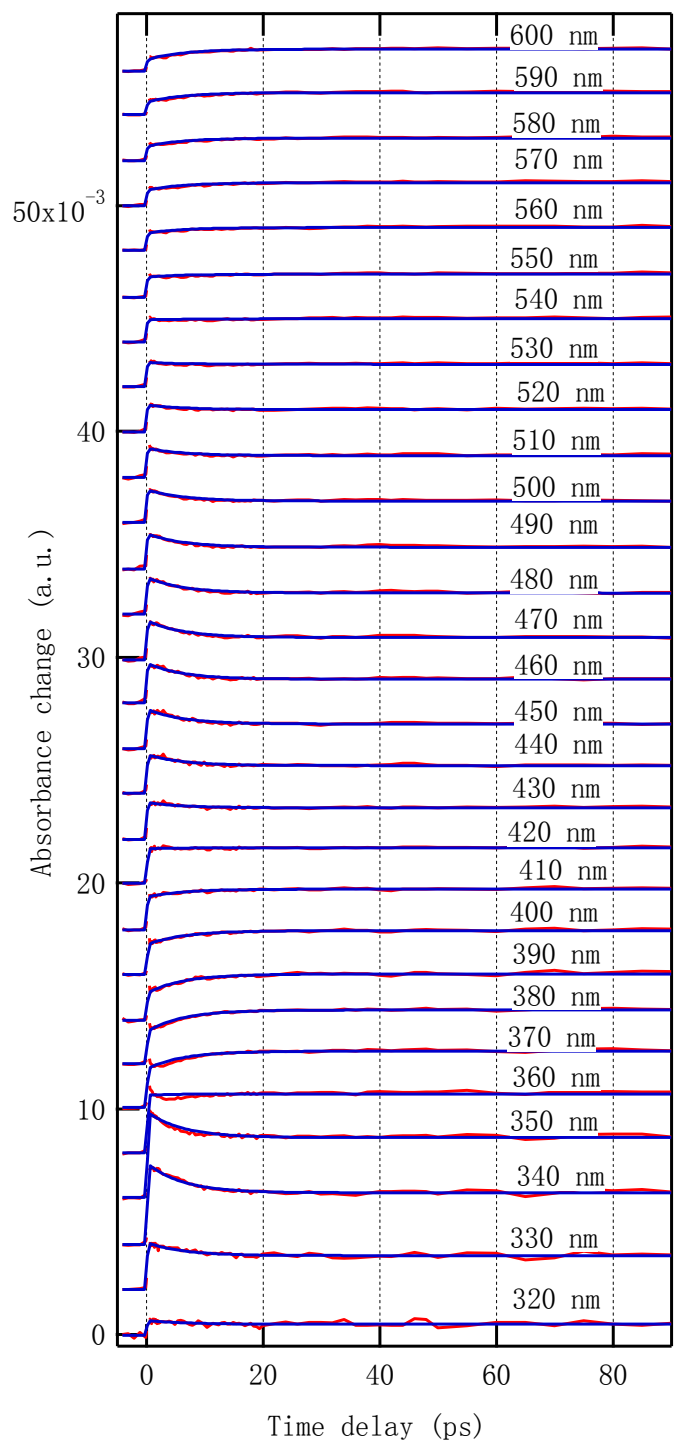

(a)

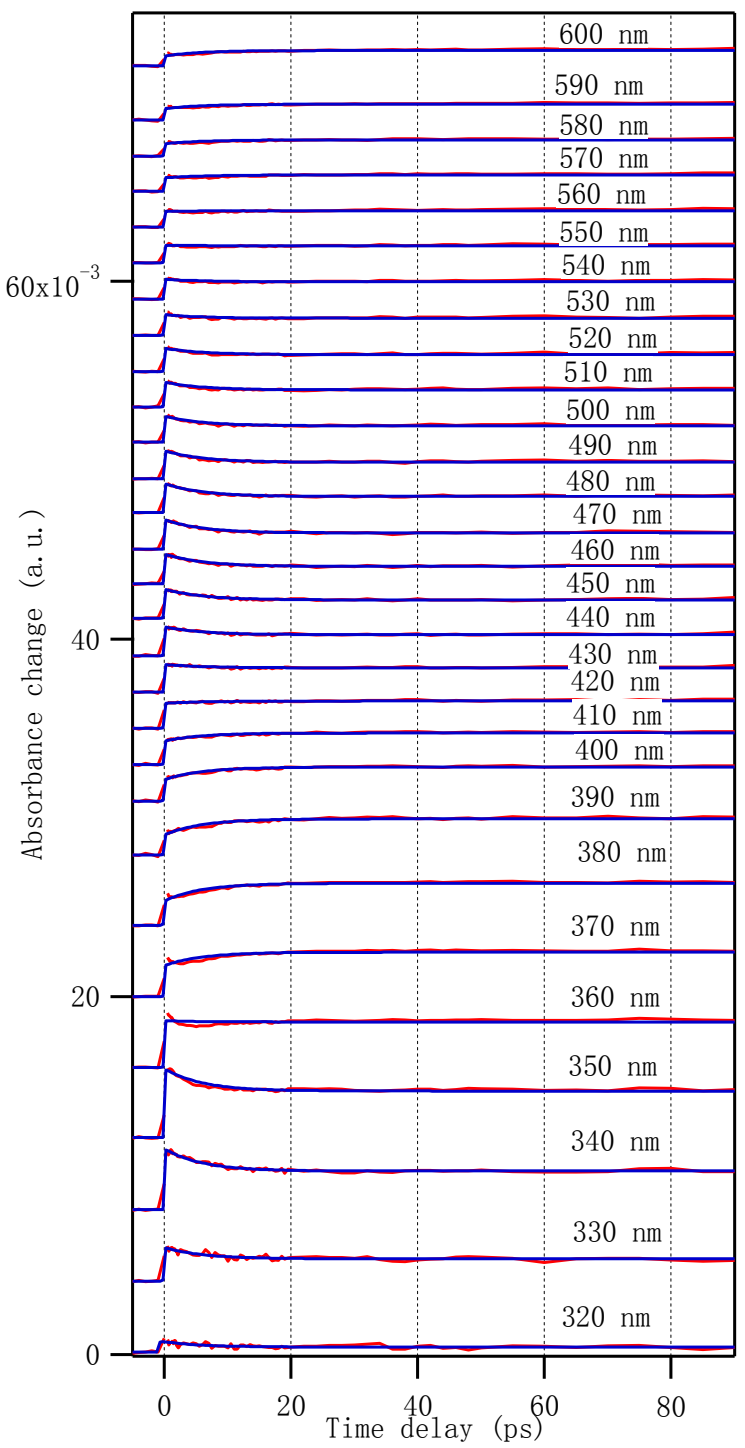

(b) 


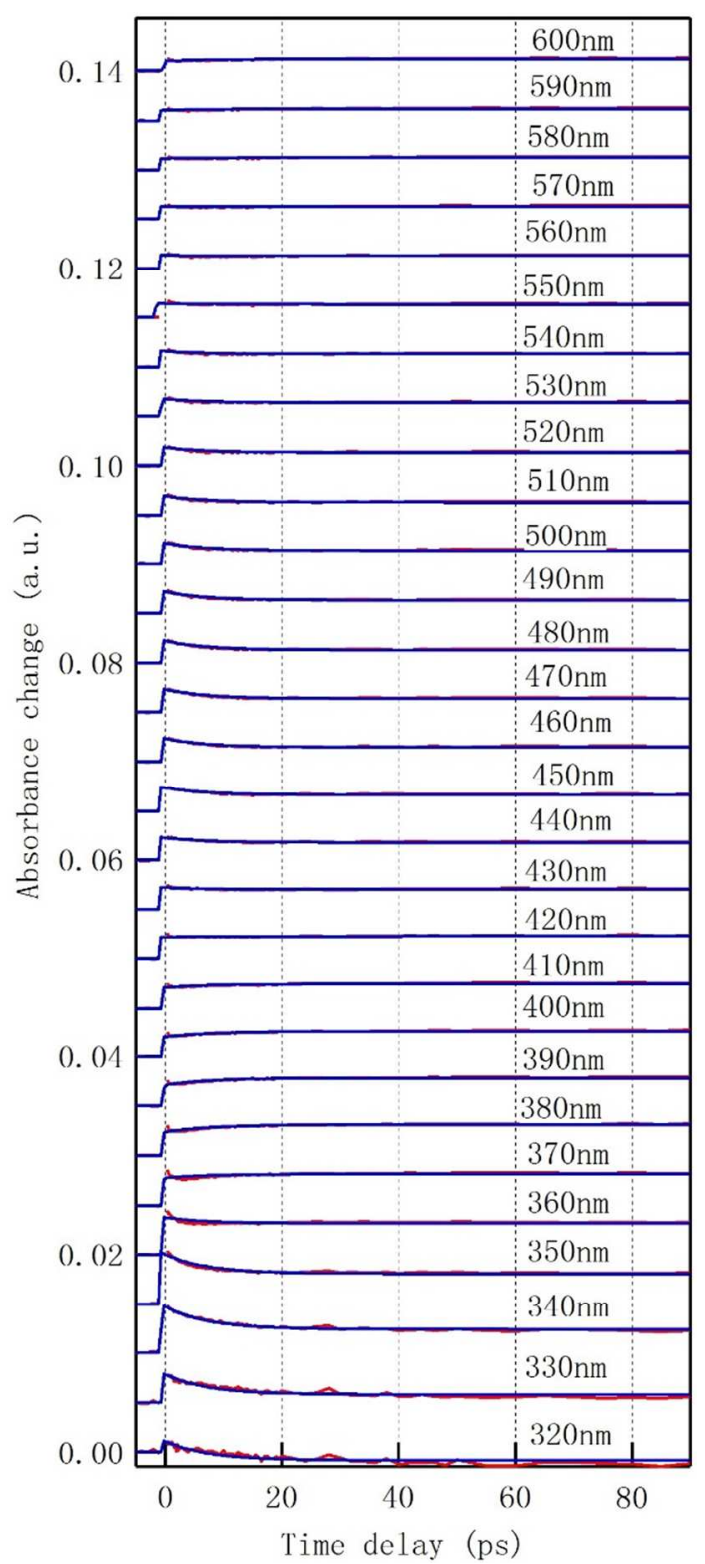

(c)

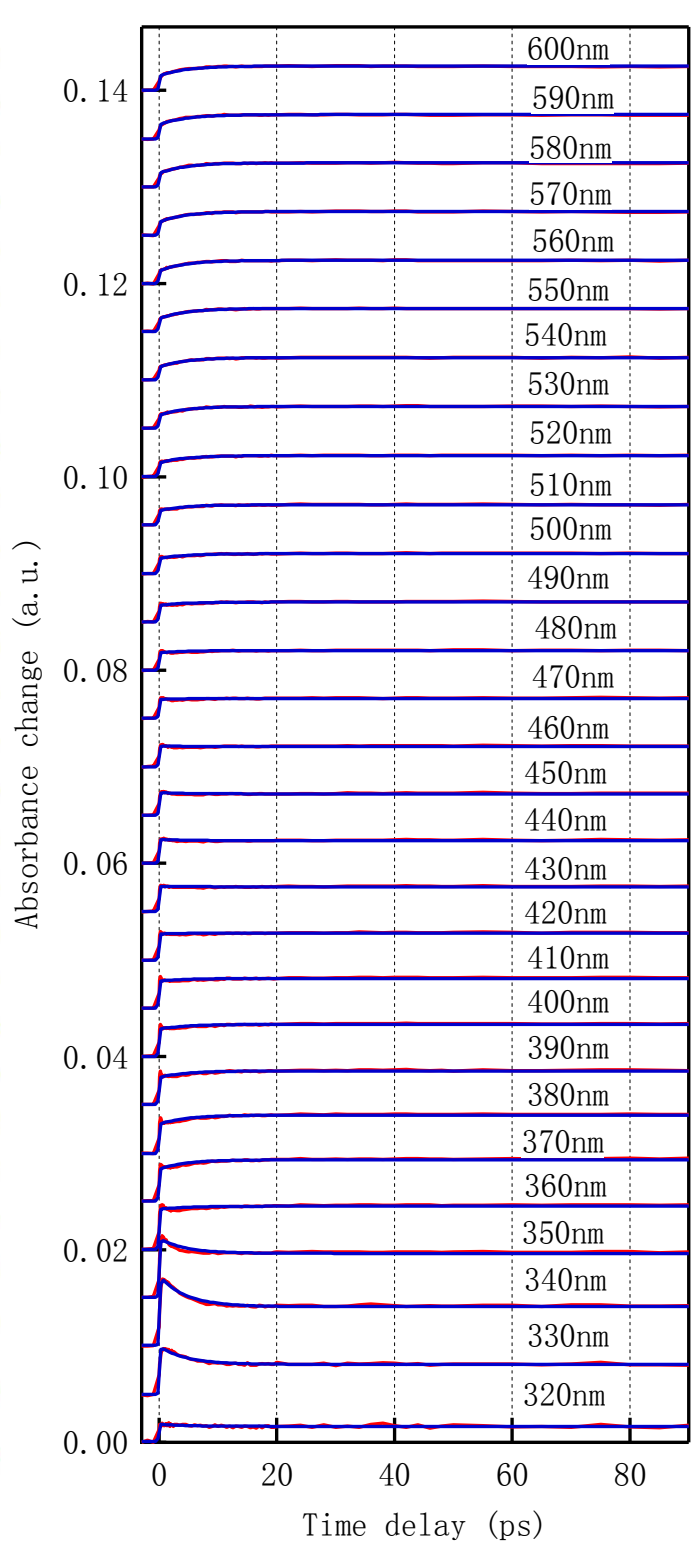

(d)

Fig. 2S Global fitting of the decay dynamics of 6-AU in methanol (a), ethanol (b), $n$-butanol (c) and water (d) at various wavelengths. Red line: experimental data; Blue line: fitting of the data. 


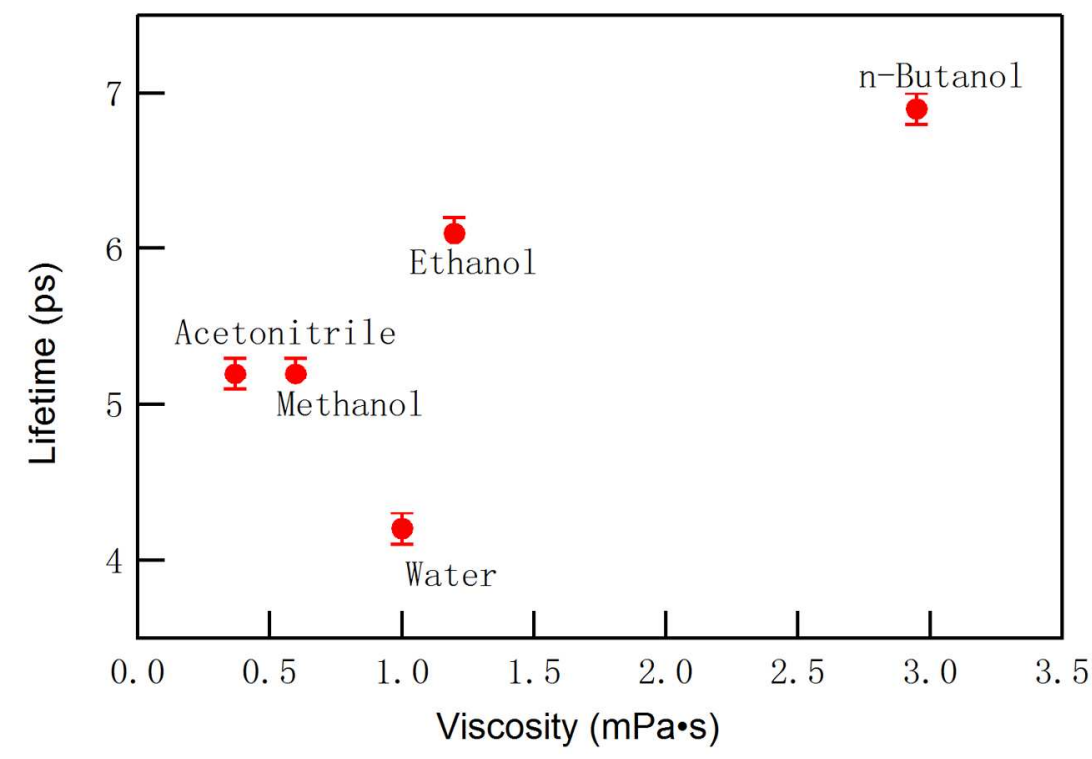

Fig. 3S Lifetime constants as a function of viscosity of different solvents. 